\title{
Structure evolution of PET under step-wise and continuous deformation modes: the effect of stress relaxation on the strain-induced morphology
}

\author{
L.V. Todorov, J.C. Viana \\ Institute for Polymers and Composites, Department of Polymer Engineering \\ University of Minho, 4800-058 Guimarães, Portugal \\ URL: $\underline{w w w . i p c . u m i n h o . p t}$ \\ e-mail: lvt@dep.uminho.pt; jcv@dep.uminho.pt
}

\begin{abstract}
This study reports about an in-situ deformation investigation under X-ray synchrotron source (Wide-Angle X-ray Scattering, WAXS) of polyethylene terephthalate (PET) following step-wise and continuous stretching modes. In this way the effect of stress relaxation on the strain-induced phase and structure evolution is investigated for PET with different initial structures: quasi-amorphous and semicrystalline. These PET initial morphologies were developed from fully amorphous PET by uniaxial stretching above the glass transition, $\mathrm{T}_{\mathrm{g}}$, and rapidly quenched. The in-situ experiments reveal significant influence of stress relaxation on the strain-induced phase and structure evolution for both PET morphologies. A relative delay in structure evolution is presented for continuous stretching because of reduced macromolecular chain relaxation during deformation. A constant increasing trend of amorphous orientation with deformation is assessed by both deformation protocols. The strain-induced phase results disclose that relaxation phenomenon contributes for: i) crystalline phase preserving during deformation for initially semi-crystalline PET; and ii) greater amorphous mass fraction for the quasi-amorphous PET.
\end{abstract}

Key words: PET, Strain-induced structure, Relaxation

\section{INTRODUCTION}

PET is a material of major commercial relevance due to its enhanced mechanical, thermal and barrier properties. These properties are greatly affected by the degree of orientation and crystallinity impart during processing. As PET can be processed by deformation in the rubbery state (e.g., stretching blow moulding) the study of the strain-induced structure development is of both academic and industrial interest. Deformation above and below $\mathrm{T}_{\mathrm{g}}$ plays an important role in the development of structure and performance. The characterization of these deformation processes is of extreme importance under processing and in-service conditions. The fast structural transition mechanisms taking place during stretching are difficult to access without proper in-situ characterization tools. The availability of synchrotron X-ray radiation made possible to measure in-situ the structural formation of PET films and fibres in real time. Many studies were conducted to investigate the effect of processing conditions and modes of deformation below [1] and above [2,3] $\mathrm{T}_{\mathrm{g}}$ from glassy PET. During uniaxial deformation above $\mathrm{T}_{\mathrm{g}}$ of amorphous PET structural development consist of three stages, according to recent models [2, 3]: i) molecular orientation occurs due to strong molecular interaction - "orientation" stage; ii) nucleus start to appear as a result of molecular orientation "nucleation" stage; and iii) crystallization develops through a growth process - "growth" stage. Three distinct PET morphologies are developed: i) an unoriented amorphous phase (isotropic); ii) a mesophase with a degree of packing order between the crystalline and the amorphous phase (anisotropic oriented amorphous phase); and iii) a crystalline phase [2, 3]. Similar structure development of initially isotropic PET was observed at temperatures slightly below $\mathrm{T}_{\mathrm{g}}$ [1]. The present study aims at investigating the structure evolution of PET with distinct initial morphologies (quasi-amorphous and 
semi-crystalline) and at evaluating the effect of relaxation phenomena on the strain-induced morphology.

\section{MATERIALS AND EXPERIMENTAL PROTOCOL}

\subsection{Materials}

The PET has an intrinsic viscosity of $0.74 \pm 0.02$ dl. $\mathrm{g}^{-1}$; a solid density of $1.40 \mathrm{~g} . \mathrm{cm}^{-3}$; an acetaldehyde level less than $1 \mathrm{ppm}$; and an average molar mass of $\mathrm{Mn} \approx 20000$ g. $\mathrm{mol}^{-1}$.

\subsection{Samples preparation}

An amorphous PET was obtained by compression moulding and rapidly quenching in the water at -5 $\pm 0.5{ }^{\circ} \mathrm{C}$. The amorphous structure was assessed by differential scanning calorimetry (DSC) and WAXS. From these plaques were cut large dumbbell-like specimens (length of $95 \mathrm{~mm}$ and cross-section $42 \times 0.3 \mathrm{~mm}$ ). Those specimens were stretched above $\mathrm{T}_{\mathrm{g}}$ in a universal testing machine Instron 4505 equipped with an environment chamber. The dumbbell-like specimens were clamped at room temperature at gauge length $55 \mathrm{~mm}$, after which the chamber was heated up by hot air to the programmed test temperature. Prior stretching each sample was allowed to thermally equilibrate for an additional $2 \mathrm{~min}$. The stretching protocol included variations of the stretching temperature, $\mathrm{T}_{\mathrm{st}}$, the stretching velocity, $\mathrm{V}_{\text {st }}$ (nominal strain-rates of 0.003 $\mathrm{s}^{-1}$ and $0.03 \mathrm{~s}^{-1}$ ) and the stretching level, $\varepsilon_{\mathrm{st}}$, according to Table 1 .

Table1. Stretching program for amorphous PET (morphological state: QA -quasi-amorphous; SC - semicrystalline).

\begin{tabular}{cccc|c}
\hline \multirow{2}{*}{ Sample } & \multicolumn{3}{c|}{ stretching parameters } & \multirow{2}{*}{ state } \\
\cline { 2 - 4 } & $\mathrm{T}_{\mathrm{st}}\left[{ }^{\circ} \mathrm{C}\right]$ & $\mathrm{V}_{\text {st }}\left[\mathrm{mm} \cdot \mathrm{min}^{-1}\right]$ & $\varepsilon_{\text {st }}$ & \\
\hline$\# 1$ & 110 & 10 & 0.60 & QA \\
$\# 2$ & 90 & 100 & 1.07 & SC \\
\hline
\end{tabular}

From the middle of the stretched specimens were cut longitudinally tensile test specimens (50 length and cross-section $4 \times 0.3 \mathrm{~mm}$ ), which were used for subsequent studies on morphology evolution during stretching at room temperature by: i) step-wise and ii) continuous stretching modes.

\subsection{Experimental protocol}

The samples were characterized in-situ by WAXS in two modes: 1) step-wise and 2) continuous stretching. These experiments were performed under synchrotron $\mathrm{CuK \alpha}$ radiation $(\lambda=0.154 \mathrm{~nm})$ at HASYLAB, DESY, Hamburg (A2 soft condensed matter beam-line). The specimens were positioned perpendicular to the incident X-ray beam with the stretching direction pointing upward. The twodimensional WAXS patterns were acquired by a MARCCD camera, and calibration was done with a crystalline PET sample. Background scattering was subtracted and all plots were normalized with respect to the incident X-ray intensity, accumulation time and specimen thickness. Experimental protocol was set as follows:

- step-wise mode: distance sample-to-detector of $135 \mathrm{~mm}$; stretching at constant crosshead velocity of $2 \mathrm{~mm} . \mathrm{min}^{-1}$; stretching was ceased for $2 \mathrm{~min}$ at constant strain; after which 2D-WAXS patterns were obtained with accumulation time of $20 \mathrm{~s}$ then stretching was restored.

- continuous stretching mode: distance sample-todetector of $14.5 \mathrm{~mm}$; stretching at constant crosshead velocity of $2 \mathrm{~mm} \cdot \mathrm{min}^{-1}$; every $60 \mathrm{~s}$ 2D-WAXS patterns were acquired with accumulation time of $20 \mathrm{~s}$.

\subsection{WAXS data analysis}

A peak-fitting program was used to deconvolute the crystalline peaks and amorphous halo from linear intensity profiles extracted from the 2D-WAXS images. The 2D-WAXS patterns treatment was adapted from the proposed by Kawakami at el. [2]. The two linear intensity profiles taken along the equatorial and meridional directions were used to estimate mass fractions of the three phases. The amount of crystalline phase was assumed to be proportional to the total area of the deconvoluted (100), (010) and (-110) peaks from the linear equatorial profile, whereas, the amount of the unoriented amorphous phase was assumed to be proportional to the area of the meridional profile. If a crystal diffraction peak was detected in the meridional scan, as the $(-103)$ reflection, its contribution was excluded. The subtraction of the crystalline and amorphous fractions from the total area in the equatorial profile was proportional to the amount of the mesophase. The mass fraction of the individual phase was taken as the ratio of the area for each phase to the total area of the equatorial profile. 
Amorphous phase orientation, $f_{a m}$, was determined by azimuthal scans obtained over $\mu=0-\pi$ integrated over a section of $2 \Theta$ values between $19.3^{\circ}$ and $20.8^{\circ}$. This annular ring is placed in between the $(010)$ and (111) crystalline reflections, an angular range where the amorphous peak was reasonably intense and had least overlap from the crystalline reflections, when present. The amorphous phase orientation, $f_{a m}$, was expressed as the Hermans' orientation factor:

$f=\frac{3\left\langle\cos ^{2} \phi\right\rangle-1}{2}$

where:

$$
\left\langle\cos ^{2} \phi\right\rangle=\frac{\int_{-\pi / 2}^{\pi / 2} I(\phi) \cos ^{2} \phi \sin \phi d \phi}{\int_{-\pi / 2}^{\pi / 2} I(\phi) \sin \phi d \phi}
$$

The $\left\langle\cos ^{2} \phi\right\rangle$ could be evaluated from the full-width at half maximum $(\Delta \phi)$ of the Gaussian azimuthal intensity distribution by evaluating the integral for each value of $\Delta \phi$ from the relation [5] :

$$
I(\phi)=\exp \left(\frac{-4 \phi^{2} \ln 2}{\Delta \phi^{2}}\right)
$$
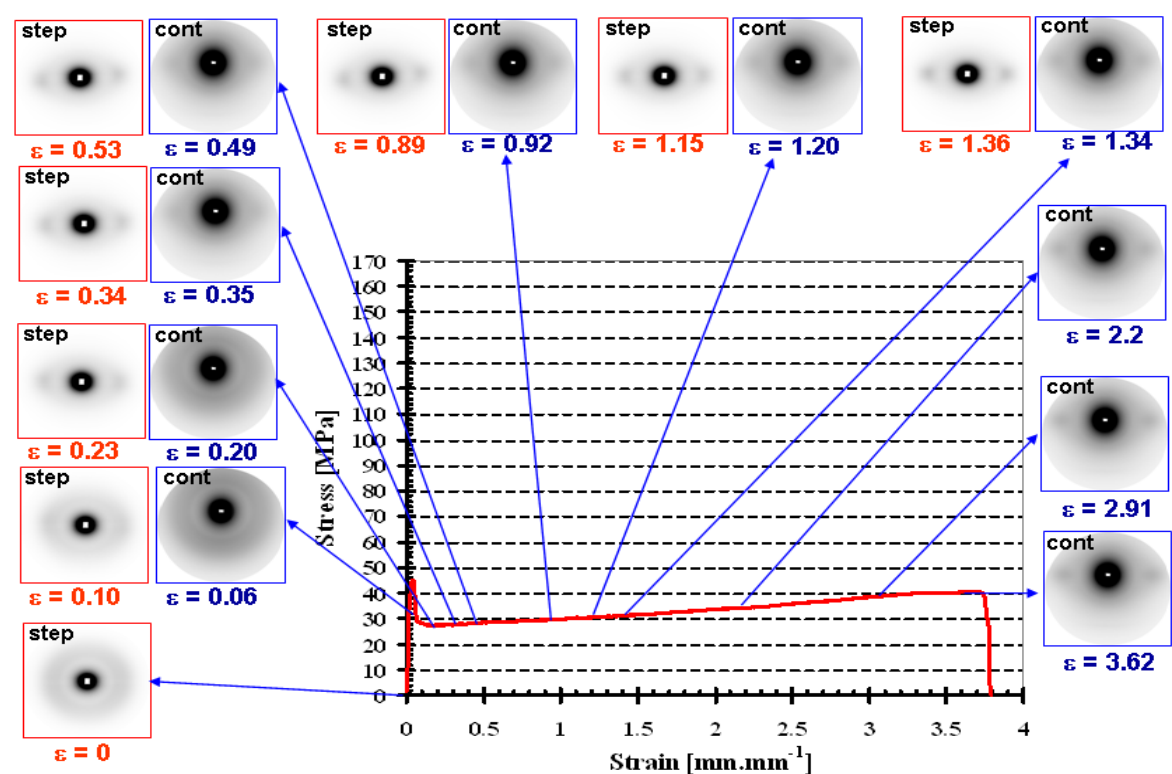

Fig. 1. Quasi-amorphous sample (\#1): stress-strain curve obtained upon continuous stretching and selected 2D WAXS patterns collected during step-wise and continuing stretching modes.

In Fig. 2 are depicted the stress-strain curve obtained upon continuous stretching of semi-crystalline sample (\#2) and selected 2D-WAXS patterns collected during step-wise and continuing stretching modes. The crystalline peak reflections on the equatorial direction are indexed as $(010),(-110)$ and (100) crystalline planes of the PET triclinic unit cell [4]. Those peaks intensify until sample breaking for step-wise mode, evidencing crystalline structure
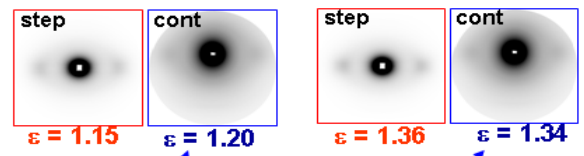

\section{RESULTS AND DISCUSSION}

Fig. 1 shows the stress-strain curve obtained during continuous stretching of amorphous sample and selected 2D-WAXS patterns collected during step-wise and continuing stretching modes. Initial pattern at zero strain indicated an amorphous halo typical of isotropic quasi-amorphous PET. When the strain reached the value of 0.23 , in case of step-wise mode the isotropic amorphous scattering ring converges into a pair of broad and intense arcs at the equator, evidencing an increment upon the inter-chain packing and the formation of a mesophase structure. In the case of continuous mode that feature become slightly pronounced at strain of 0.35 . This (delay) difference in the morphology development is revealed until the end of stretching and may be attributed to chain relaxation phenomena occurring for the step-wise mode. The absence of crystalline peaks reflections in all patterns along the stretching indicates that amorphous phase transforms into more oriented mesophase, but a crystalline structure is not developed.

preservation. In the case of continuous stretching, the crystalline peak reflections are weaker and they are converted into intensified spots on the equator $(\varepsilon=0.20)$, suggesting some destruction of crystalline phase and its alteration into a mesophase during strain progress. The crystalline structure shown by the step-wise mode must then be formed during the interrupted deformation stage, as consequence of chain relaxation. 


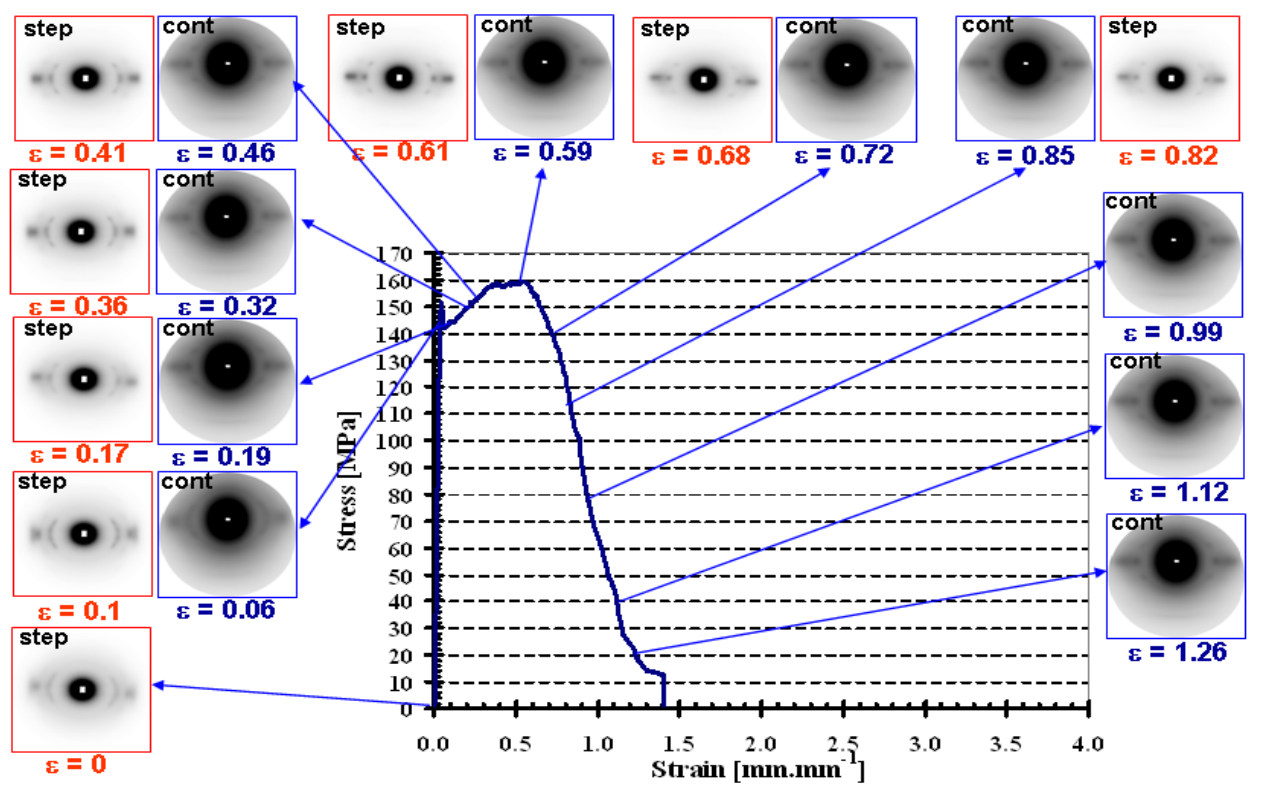

Fig. 2. Semi-crystalline sample (\#2): stress-strain curve obtained upon continuous stretching and selected 2D WAXS patterns collected during step-wise and continuing stretching modes.

Fig. 3 shows the evolution of amorphous and mesophase mass fractions during step-wise and continuous stretching modes of the quasi-amorphous PET. For step-wise stretching mode, mass fractions of amorphous and mesophase remain constant until $\varepsilon \approx 0.3$. Afterwards, the amorphous fraction decreased dramatically and the mesophase fraction rapidly expands. Upon higher deformation levels, there is only a slight increment upon the mesophase contents at the expenses of a reduction on the isotropic amorphous phase. In the case of continuous stretching, immediately after the yield point is observed a drastic reduction of amorphous phase and respective transformation into a highly oriented mesophase.

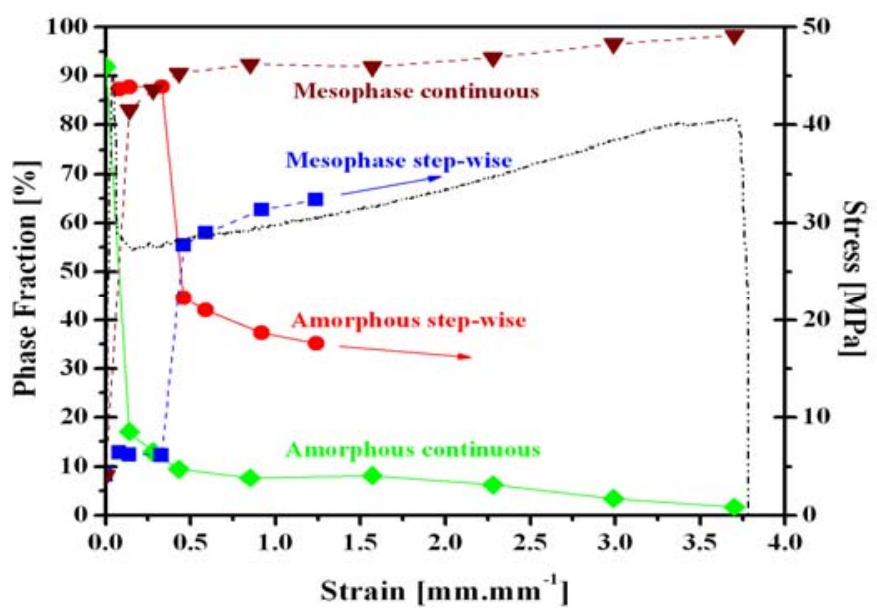

Fig. 3. Evolution of amorphous and mesophase fractions of quasi-amorphous sample (\#1) upon step-wise and continuous stretching (stress-strain curve also shown).

Fig.4 presents the evolution of phase's fractions during stretching for semi-crystalline sample (\#2). For continuous stretching, immediately after yielding is observed an increment on the mesophase and correspondent diminishing of amorphous phase. Concomitantly, there is a reduction on the crystalline phase content. At $\varepsilon \approx 0.3$, no more crystalline phase is detected, as consequence of its destruction during stretching at constant velocity. After that strain level, the amounts of amorphous and mesophase are kept approximately constant until sample breakage.

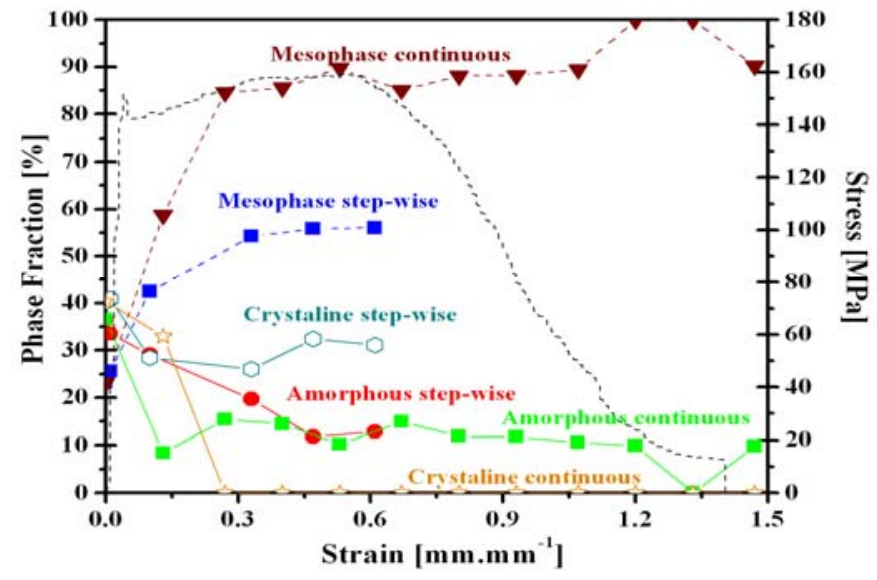

Fig. 4. Evolution of amorphous, mesophase and crystalline fractions of semi-crystalline PET (\#2) upon step-wise and continuous stretching (stress-strain curve also shown).

The step-wise mode led to a sudden transition of amorphous into oriented mesophase with a weak decrease in the crystalline phase fraction. During stretching interruption, chain relaxation allows for crystallization of PET. It can be argued that still occurs destruction of the crystalline phase upon stretching, but then a re-crystallization occurs during the stopped period.

Amorphous phase orientation of both samples (\#1 and \#2) and modes are presented in Fig. 5. For 
quasi-amorphous PET (\#1), the amorphous phase orientation increases during stretching under both deformation protocols. Semi-crystalline PET (\#2) shows already a high level of amorphous phase orientation before deformation. It seems that the amorphous phase is highly oriented between the crystalline structures. Upon step-wise stretching, there is an increment of amorphous orientation with strain. The crystalline phase developed make difficult the relaxation of the highly oriented mesophase upon interrupted stretching. However, during continuous mode deformation the level of amorphous phase orientation remains almost constant. It slightly decreases for the initial strain levels. This may suggest that the destruction of the crystalline structure is accompanied by some relaxation of the amorphous phase. Afterwards $(\varepsilon>0.3)$, the level of amorphous orientation slightly increase, remaining thereafter constant. Interestingly, the final levels of amorphous orientation are similar for both deformation modes regardless the initial PET structure. Furthermore, they are always higher for step-wise deformation that also achieved low deformation levels. This enhanced orientation level may be attributed to a better re-arrangement of the mesophase during the relaxation over the interrupted deformation period.

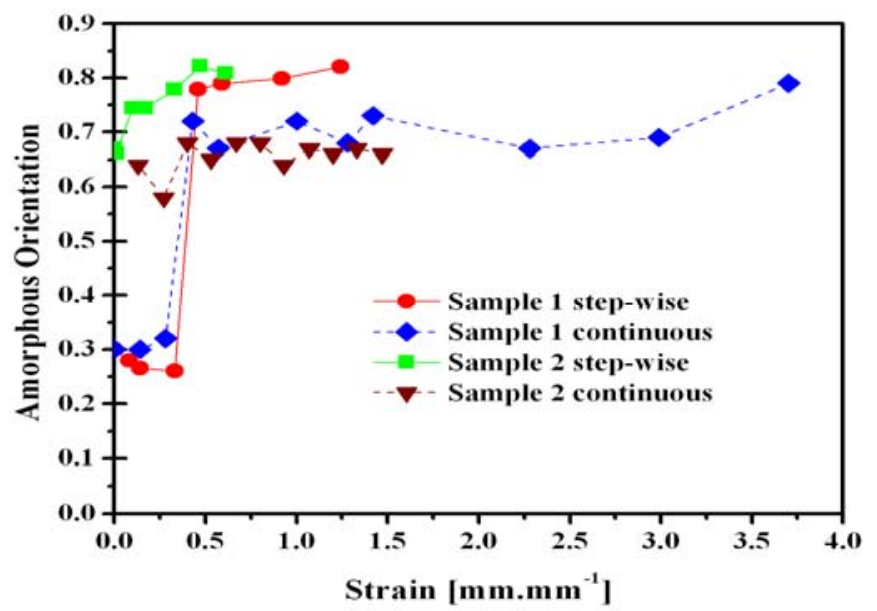

Fig. 5. Amorphous phase orientation for quasi-amorphous (\#1) and semi-crystalline (\#2) PET upon step-wise and continuing stretching modes.

\section{CONCLUSIONS}

Under the conditions of this study, initially quasiamorphous PET develops under deformation a more organised and oriented mesophase, without vestiges of a crystalline structure, by gradual transformation of isotropic amorphous phase. Chain relaxation during step-wise deformation contributes for a greater amount of mesophase, suggesting some macromolecular re-arrangement during interrupted deformation. For initially semi-crystalline PET, there is also a development of a highly oriented mesophase upon stretching and a decrement upon the amount of crystalline phase during stretching. In the case of step-wise deformation, the amount of crystalline phase remains constant, suggesting that polymer relaxation promotes the development for crystalline phase by re-crystallization during interrupted deformation stage. This degree of crystallinity imparts a reduced deformability of the PET. Upon step-wise stretching, the level of orientation of amorphous phase increases with deformation, but more significantly for initially quasi-amorphous PET. For semi-crystalline PET, continuous stretching result in a slight decrease of amorphous phase orientation for the initial strain levels, accompanying the decrement of crystallinity. After complete destruction of this crystalline structure, amorphous phase orientation increase slightly and it is kept constant thereafter. For both PET morphologies, the step-wise deformation resulted in higher levels of amorphous phase orientation that was attributed to a better rearrangement of the mesophase during the relaxation over the interrupted deformation period.

\section{ACKNOWLEDGEMENTS}

This work was partially supported by DESY and the European Commission under HASYLAB Project DESY-D-II-05-101 EC and the FP6 contracts RII3-CT-2004-506008 (IA-SFS) and SRTP-505204-1 (project APT_PACK), and FCT MICROTEST-POCTI/CTM/46940/2002.

\section{REFERENCES}

1. S. Ran, Z. Wang, C. Burger, B. Chu, B.S. Hsiao, Macromolecules 35 (2002) 10102-07.

2. D. Kawakami, B.S. Hsiao, S. Ran, C. Burger, B. Fu, I. Sics, B. Chu, and T. Kikutani, Polymer, 45 (2004) 90518.

3. D. Kawakami, S. Ran, C. Burger, C. Avila-Orta, I. Sics, B. Chu, S.H. Benjamin, T. Kikutani, Macromolecules, 39 (2006) 2909-20.

4. U. Goschel, K. Deutschert, V. Abetzt, Polymer, 37 (1996) 1-6.

5. E.B. Gowd, C. Ramesh, M.S. Byrne, N.S. Murthy, J. Radhakrishnan, Polymer, 45 (2004) 6707-12. 